will likely be required to definitively elucidate how $\mathrm{P}_{2} \mathrm{Y}_{14}$ mediates the HSPC stress response $(10,22)$. If $P Y 2_{14}$-deficient HSCs within the animal model system developed by Cho, Yusuf, and colleagues do indeed prove to be functionally senescent, then this could be an exciting model system for studying the onset of stress-induced senescence within the HSC compartment. A better understanding of the regulators of HSC stress response has important implications for regenerative medicine.

\section{Acknowledgments}

D.J. Rossi is a New York Stem Cell Foundation Robertson Investigator.

Address correspondence to: Derrick J. Rossi, 200 Longwood Ave., Warren Alpert Building, Room \#149e, Boston, Massachusetts 02115, USA. Phone: 617.713.8900; Fax: 617.713.8910; E-mail: derrick.rossi@ childrens.harvard.edu.

\footnotetext{
1. Hayflick L, Moorhead PS. The serial cultivation of human diploid cell strains. Exp Cell Res. 1961;25:585-621.

2. Campisi J, d'Adda di Fagagna F. Cellular senescence: when bad things happen to good cells. Nat Rev Mol Cell Biol. 2007;8(9):729-740.
}

3. Kuilman T, Michaloglou C, Mooi WJ, Peeper DS. The essence of senescence. Genes Dev. 2010; 24(22):2463-2479.

4. Salama R, Sadaie M, Hoare M, Narita M. Cellular senescence and its effector programs. Genes Dev. 2014;28(2):99-114.

5. Rossi DJ, et al. Cell intrinsic alterations underlie hematopoietic stem cell aging. Proc Natl Acad Sci US A. 2005;102(26):9194-9199.

6. Dykstra B, Olthof S, Schreuder J, Ritsema M, de Haan G. Clonal analysis reveals multiple functional defects of aged murine hematopoietic stem cells. J Exp Med. 2011;208(13):2691-2703

7. Beerman I, et al. Functionally distinct hematopoietic stem cells modulate hematopoietic lineage potential during aging by a mechanism of clonal expansion. Proc Natl Acad Sci U S A. 2010; 107(12):5465-5470

8. Pang WW, et al. Human bone marrow hematopoietic stem cells are increased in frequency and myeloidbiased with age. Proc Natl Acad Sci U S A. 2011; 108(50):20012-20017.

9. Rossi DJ, Bryder D, Seita J, Nussenzweig A, Hoeijmakers J, Weissman IL. Deficiencies in DNA damage repair limit the function of haematopoietic stem cells with age. Nature. 2007 447(7145):725-729.

10. Beerman I, Seita J, Inlay MA, Weissman IL, Ross DJ. Quiescent hematopoietic stem cells accumulate DNA damage during aging that is repaired upon entry into cell cycle. Cell Stem Cell. 2014;pii:S19345909(14)00153-2.

11. Florian MC, et al. Cdc42 activity regulates hematopoietic stem cell aging and rejuvenation. Cell Stem Cell. 2012;10(5):520-530.

12. Beerman I, et al. Proliferation-dependent alterations of the DNA methylation landscape underlie hematopoietic stem cell aging. Cell Stem Cell. 2013; 12(4):413-425.

13. Sun D, et al. Epigenomic profiling of young and aged HSCs reveals concerted changes during aging that reinforce self-renewal. Cell Stem Cell. 2014; 14(5):673-688.

14. Sperka T, Wang J, Rudolph KL. DNA damage checkpoints in stem cells, ageing and cancer. Nat Rev Mol Cell Biol. 2012;13(9):579-590.

15. Attema JL, Pronk CJ, Norddahl GL, Nygren JM, Bryder D. Hematopoietic stem cell ageing is uncoupled from p16 INK4A-mediated senescence. Oncogene. 2009;28(22):2238-2243.

16. Janzen V, et al. Stem-cell ageing modified by the cyclin-dependent kinase inhibitor p16INK4a. Nature. 2006;443(7110):421-426

17. Cho J, et al. Purinergic $\mathrm{P}_{2} \mathrm{Y}_{14}$ receptor modulates stress-induced hematopoietic stem/progenitor cell senescence. J Clin Invest. 2014;124(7):3159-3171.

18. Ito $\mathrm{K}$, et al. Regulation of reactive oxygen species by Atm is essential for proper response to DNA double-strand breaks in lymphocytes. J Immunol. 2007; 178(1):103-110.

19. Tothova $Z$, et al. FoxOs are critical mediators of hematopoietic stem cell resistance to physiologic oxidative stress. Cell. 2007;128(2):325-339.

20. Miyamoto K, et al. Foxo3a is essential for maintenance of the hematopoietic stem cell pool. Cell Stem Cell. 2007;1(1):101-112.

21. Yalcin S, et al. Foxo3 is essential for the regulation of ataxia telangiectasia mutated and oxidative stress-mediated homeostasis of hematopoietic stem cells. J Biol Chem. 2008;283(37):25692-25705.

22. Shao L, et al. Total body irradiation causes longterm mouse BM injury via induction of HSC premature senescence in an Ink4a- and Arf-independent manner. Blood. 2014;123(20):3105-3115

\title{
Twisting mice move the dystonia field forward
}

\author{
Åsa Petersén ${ }^{1}$ and Deniz Kirik² \\ ${ }^{1}$ Translational Neuroendocrine Research Unit and ${ }^{2}$ Brain Repair and Imaging in Neural Systems (B.R.A.I.N.S.) Unit, \\ Department of Experimental Medical Sciences, Lund University, Lund, Sweden.
}

\begin{abstract}
A common form of the hyperkinetic movement disorder dystonia is caused by mutations in the gene TOR1A (located within the DYT1 locus), which encodes the ATPase torsinA. The underlying neurobiological mechanisms that result in dystonia are poorly understood, and progress in the field has been hampered by the absence of a dystonia-like phenotype in animal models with genetic modification of Tor1a. In this issue of the JCI, Liang et al. establish the first animal model with a dystonic motor phenotype and link torsinA hypofunction to the development of early neuropathological changes in distinct sensorimotor regions. The findings of this study will likely play an important role in elucidating the neural substrate for dystonia and should stimulate systematic neuropathological and imaging studies in carriers of TOR $1 A$ mutations.
\end{abstract}

\section{Neurological disorders and the need for animal models}

For many brain disorders, identification and characterization of the underlying

Conflict of interest: Deniz Kirik receives financial compensation in the form of retainer fees, milestones, and royalty payments from Genepod Therapeutics $A B$ and receives research grant support from ParkCell AB.

Citation for this article: J Clin Invest. 2014;

124(7):2848-2850. doi:10.1172/JCI76624. neurobiological mechanisms remains a challenge for clinicians and scientists. Lack of defined neural substrates and an understanding of the pathways responsible for neurological and psychiatric symptoms has limited the development of novel therapies, which are urgently needed to improve the care and quality of life of affected individuals. In this issue, Liang and collaborators present animal models that recapitulate the major clinical symptomatology of dystonia (1). The study by Liang and colleagues represents an important leap forward for the dystonia research field.

\section{Dystonia: the twists and turns}

Dystonia is characterized by sustained or intermittent muscle contractions that cause abnormal, often repetitive, twisting movements and postures and is now recognized as a heterogenous group of hyperkinetic movement disorders. The term dystonia was coined in 1911 by Herman Oppenheim, who used "dystonia musculorum deformans" to describe a childhoodonset form of generalized dystonia (2). These disorders have traditionally been classified as either primary or secondary dystonias. Primary dystonia is considered to only present with tremor or myoclonus as an additional neurological symptom 
in the absence of any neuropathological changes, whereas secondary dystonia is considered the consequence of a hereditary neurodegenerative disorder or an insult to the brain, and other neurological symptoms can be manifest. More recently, the dystonia classification system has been called into question. In particular, the division into primary and secondary dystonia has been criticized as neuroimaging and neuropathological studies have begun to indicate the presence of structural changes in primary dystonia (3). The recent international consensus classification has instead proposed to use a two-axes classification based on clinical characteristics and etiology (4).

There is a considerable genetic contribution for many forms of dystonia. The first dystonia locus (DYT1) was identified in 1990 and localized to chromosome 9 (5). The gene, which was identified seven years later and named TOR1A, encodes for the ubiquitously expressed torsin A protein (6). Torsin $\mathrm{A}$ is a member of the $\mathrm{AAA}^{+}$family of ATPases present in the endoplasmic reticulum/nuclear envelope space and is thought to play a role in structural integrity and protein trafficking in the cell (reviewed in ref. 7). The DYT1/TOR1A mutation is responsible for around 50\% of early onset primary dystonia cases (8). Most often, the genetic mutation in DYT1-related dystonia is an in-frame GAG deletion (termed $\Delta \mathrm{E}$ mutant), which is thought to result in at least a partial loss of torsinA function. The penetrance of the mutation is around $30 \%$ to $40 \%$, and in these patients the symptoms are variable, ranging from a mild focal presentation to disabling generalized dystonia (8). Although a few of the available studies suggest the presence of rather unspecific structural and morphological alterations in the substantia nigra and the cerebellum $(9,10)$, there is a general paucity of more comprehensive clinical studies examining structural changes in the brain of DYT1dystonia patients.

\section{A dystonia model with the right moves}

Studies of animals with genetic modification of the Dyt1/Tor1a gene have suffered from the inability of these models to display overt dystonia; therefore, these preclinical models lack validity for studying overt clinical symptoms. Previous models have been engineered with a heterozygous knockin of the Tor1a $\Delta \mathrm{E}$ mutation or with brain region-specific knockout of the Dyt1 locus; however, these mutations do not result in a clear dystonic motor phenotype (recently reviewed in ref. 11). On the other hand, homozygous knockout of Tor1A or homozygous knockin of the Torla $\Delta \mathrm{E}$ mutation in mice results in neonatal lethality, and therefore, these models are not useful (12). Based on previous attempts to develop a murine dystonia model, Liang and collaborators hypothesized that a dystonia mouse would require torsin A deficiency in critical brain areas during postnatal CNS development in order to develop a clinically relevant phenotype. Toward this goal, Liang and colleagues generated mice that lacked the Torla gene in the entire CNS and found that these mice developed striking abnormal twisting movements that are indicative of dystonia. The severe early-onset phenotype observed in mice lacking torsinA in the CNS was accompanied by premature death at postnatal day 16. Liang et al. took their dystonia mouse model one step further and created mice that are heterozygous for the Tor1a $\Delta \mathrm{E}$ mutation and a floxed WT Torla allele, which can be deleted in select tissues, allowing expression of torsin $\mathrm{A} \Delta \mathrm{E}$ in the absence of the WT protein in the CNS. Liang et al. elegantly demonstrate that these animals develop a dystonia-like phenotype as early as the second postnatal week, but survive to adulthood. Interestingly, the CNS-specific Tor 1a $\Delta \mathrm{E}$ mutant mice showed an age-related improvement in behavioral phenotypes, suggesting that the residual function of torsinA may partly compensate for the early deficits in the maturing CNS (1).

Importantly, the behavioral abnormalities in the mouse models developed by Liang and colleagues were accompanied by perinuclear accumulation of ubiquitin as well as neuropathological changes, including gliosis, caspase- 3 activation, and ER stress in selective sensorimotor regions. Neuropathology was observed in deep layers of sensorimotor cortex, ventral posterior thalamus, globus pallidus, deep cerebellar nuclei, red nucleus, and the facial nerve nuclei. Thus, a second and equally important aspect of the study by Liang and colleagues is the linkage of neuropathological changes in the brain to a model of "primary" dystonia, which provides further support in the debate on the presence of structural brain changes in this disease. Although Liang and collaborators detected a selec- tive vulnerability of certain sensorimotor cells to torsinA deficiency, further neuropathological analyses will be important to determine the full extent of structural and morphological changes in these models. Nevertheless, the findings by Liang et al. call for further systematic analyses of postmortem tissue from individuals with DYT1-related dystonia in order to elucidate the level of neuropathology in the clinical setting.

\section{Conclusions and future directions}

The development of animal models for the study of brain disorders in general has been important for the progress of neuroscience research. Initially, nonspecific lesions in different brain areas of mice helped lead to knowledge about their function. More specific neurotoxic lesions mimicked neuropathological changes observed in clinical conditions and provided a basis both for testing hypotheses related to mechanisms of disease and evaluating restorative therapies. With the identification of the genetic causes of neurodegenerative disorders, the generation of animal models with better construct validity has been possible; however, studies in mice that replicate the genetic mutations identified in patients have seldom resulted in an exact phenocopy of clinical symptoms. In many instances, it appears that the genetic burden in an animal model needs to be exaggerated in order to produce a robust and clinically relevant phenotype. In the case of dystonia, Liang and collaborators engineered mice with alterations of the Dyt1/Torla gene that are more severe than what is present in patients and therefore compromised construct validity in favor of the face validity (1). A similar approach to murine model development and analysis has also been beneficial for Huntington disease (HD), a neurodegenerative movement disorder caused by an expanded CAG repeat in the gene coding for the huntingtin protein (13). In the case of HD, the animal models with severe phenotypes were obtained only when mice were engineered to express either a mutant HD protein with a much longer polyglutamine stretch than seen in any patients or a truncated and more toxic form of the protein (14). Nevertheless, the similarities found between clinical manifestations in murine models and patients, such as in the HD field, support the value of these models for examining 
potential disease mechanisms as well as for testing therapeutic strategies (15). Novel and sometimes unexpected discoveries made in animal models often become the foundation for studies using patient material, which can confirm the clinical relevance of the findings. Availability of a wide range of animal models with different advantages and varying degrees of validity in different domains directly affects the pace of advancement in translational and clinical research for diseases of the brain. The mouse models produced by Liang and collaborators are likely to play an important role in these endeavors in the dystonia research field.

\section{Acknowledgments}

D. Kirik is supported by the Swedish Research Council (2008-3092, 20122586,2012-5854) and a European Research Council ERC Starting Grant (TreatPD, 242932). Å. Petersén is sup- ported by the Swedish Research Council (2013-3537).

Address correspondence to: Deniz Kirik, B.R.A.I.N.S. Unit, BMC D11, 22184 Lund, Sweden. Phone: 46.46.2220564; Fax: 46.46.2223436; E-mail: deniz.kirik@ med.lu.se.

1. Liang C-C, Tanabe LM, Jou S, Chi F, Dauer WT. TorsinA hypofunction causes abnormal twisting movements and sensorimotor circuit neurodegeneration. J Clin Invest. 2014;124(7):3080-3092.

2. Oppenheim H. Ueber eine eigenartige Krampfkrankheit des kindlichen und jugendlichen Alters (dysbasia lordotica progressiva, dystonia musculorum deformans) Neurol Centralbr. 1911;75:323-345.

3. Standaert DG. Update on the pathology of dystonia. Neurobiol Dis. 2011;42(2):148-151.

4. Albanese A, et al. Phenomenology and classification of dystonia: a consensus update. Mov Disord. 2013; 28(7):863-873.

5. Kramer PL, et al. Dystonia gene in Ashkenazi Jewish population is located on chromosome 9q32-34. Ann Neurol. 1990;27(2):114-120.

6. Ozelius LJ, et al. The early-onset torsion dystonia gene (DYT1) encodes an ATP-binding protein. Nat
Genet. 1997;17(1):40-48.

7. Granata A, Schiavo G, Warner TT. TorsinA and dystonia: from nuclear envelope to synapse. J Neurochem. 2009;109(6):1596-1609.

8. Charlesworth G, Bhatia KP, Wood NW. The genetics of dystonia: new twists in an old tale. Brain. 2013; 136(pt 7):2017-2037.

9. Rostasy K, et al. TorsinA protein and neuropathology in early onset generalized dystonia with GAG deletion. Neurobiol Dis. 2003;12(1):11-24.

10. Argyelan M, et al. Cerebellothalamocortical connectivity regulates penetrance in dystonia. J Neurosci. 2009; 29(31):9740-9747.

11. Oleas J, Yokoi F, Deandrade MP, Pisani A, Li Y. Engineering animal models of dystonia. Mov Disord. 2013;28(7):990-1000.

12. Goodchild RE, Kim CE, Dauer WT. Loss of the dystonia-associated protein torsinA selectively disrupts the neuronal nuclear envelope. Neuron. 2005; 48(6):923-932.

13. The Huntington's Disease Collaborative Research Group. A novel gene containing a trinucleotide repeat that is expanded and unstable on Huntington's disease chromosomes. Cell. 1993;72(6):971-983.

14. Pouladi MA, Morton AJ, Hayden MR. Choosing an animal model for the study of Huntington's disease. Nat Rev Neurosci. 2013;14(10):708-721.

15. Crook ZR, Housman D. Huntington's disease: can mice lead the way to treatment? Neuron. 2011; 69(3):423-435.

\title{
Cardiac fibroblasts in pressure overload hypertrophy: the enemy within?
}

\author{
Nenad Bursac \\ Department of Biomedical Engineering, Duke University, Durham, North Carolina, USA.
}

\begin{abstract}
Cardiac fibroblasts have been long recognized as active participants in heart disease; however, their exact physiological and pathological roles remain elusive, mainly due to the lack of specific markers. In this issue of the JCI, Moore-Morris and colleagues used a fibroblast-specific collagen1a1-GFP reporter to demonstrate that fibroblast accumulation after aortic banding in murine hearts arises almost exclusively from proliferation of resident fibroblasts originating from both the epicardium and a previously unrecognized source, the endocardium. Further characterization of fibroblast origin and function in different types and stages of heart disease could lead to development of improved fibroblast-targeted cardiac therapies.
\end{abstract}

\section{Lack of specific cardiac fibroblast markers}

Cardiac fibroblasts comprise 30\% to $70 \%$ of all the cells in the healthy adult heart (1). The number of fibroblasts in the heart is not constant and changes dynamically with development, disease, and aging (2, 3). Traditionally, cardiac fibroblasts have been thought to play passive roles in the heart and to be solely responsible for

Conflict of interest: The author has declared that no conflict of interest exists.

Citation for this article: J Clin Invest. 2014; 124(7):2850-2853. doi:10.1172/JCI76628. maintaining homeostasis of extracellular matrix proteins, including type I and III collagens and fibronectin. Due to their ubiquitous presence in the heart, fibroblasts are well poised to actively regulate and modify cardiac function through their direct contacts with other cardiac cells and matrix as well as through secretion of different cytokines, matrix proteins, and proteases. Over the last decade, the pleiotropic roles of fibroblasts in cardiac biology and disease have been studied extensively (reviewed in refs. 4, 5); however, the lack of specific and comprehensive markers of fibroblast pheno- types has hampered the progress in this important research field. In particular, vimentin, the most inclusive marker of cardiac fibroblasts, also labels all other mesoderm-derived cells in the heart. Thymus cell antigen-1 (Thy-1, also known as CD90), discoidin domain receptor-2 (DDR2), prolyl-4-hydroxylase (P4H), transcription factor 21 (TCF21, also known as epicardin, Pod1, and capsulin), periostin, cadherin-11, and fibroblast-specific protein-1 (FSP1, also known as S100A4) have all been used to study cardiac fibroblasts, but all of these markers label only a subset of fibroblasts, have poor expression in the healthy adult heart, or nonselectively label endothelial, smooth muscle, or immune/ inflammatory cells (6). Because the pathological tissue remodeling that is secondary to cardiac injury and inflammation involves contributions from both resident and extracardiac cells, the lack of adequate markers for cardiac fibroblasts may lead to erroneous conclusions about their origin, roles, and potential to be therapeutically targeted in fibrotic heart disease. 